\title{
Effects of the Shape of the Base of Support and Dual Task Execution on Postural Control
}

\author{
Yungon Lee', Sunghoon Shin ${ }^{1 *}$ \\ ${ }^{1}$ School of Kinesiology, Yeungnam Univeristy, Gyeongsan, South Korea
}

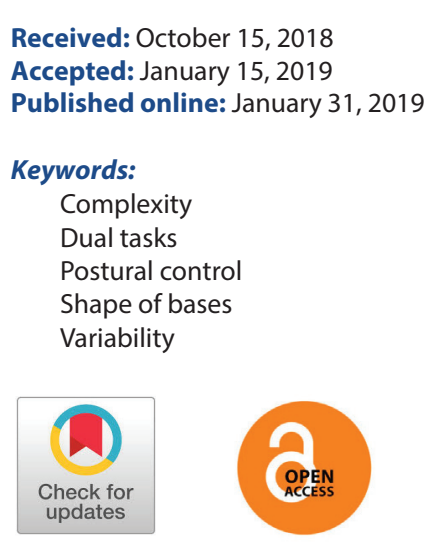

\section{ABSTRACT}

OBJECTIVES The current investigation examined the effects of a dual task execution, based on the shape of the base of support (BOS) and the task characteristics (i.e., dual or non-dual tasks), on the posture control, and how the interaction effects appear according to the combination of the two variables.

METHODS A total of 22 young adults ( 11 males and 11 females) participated in the study. The participants were instructed to stand quietly based on the shape of their BOS (normal stance, semi-tandem, or tandem) on two left and right force plates. For the dual tasks, an inverse calculation in which the subjects quietly stated to the examiner three-digit numbers (between 300 and 900), after subtracting them by 7 , was conducted. A two-way repeated measures ANOVA with two within factors was used. The dependent variables were set as the linear parameters (mean velocity (MV), RMS, and 95\% ellipse sway area (CEA)), power frequency parameters (95\% power frequency density (F95) and mean frequency (MF)), and complexity parameters (sample entropy (SampEn) and fractal dimension (FD)) of the center of pressure (COP) time series at each anteroposterior (AP) and mediolateral (ML) direction.

RESULTS For the linear parameters, RMS_AP and CEA had an interaction effect on the shape of the BOS and the presence of dual tasks $(p<.05)$. For the frequency parameters, F95_AP and MF_AP had an interaction effect on the shape of the BOS and the presence or absence of dual tasks $(p<.05)$. For the nonlinear parameters, SampEn_AP and FD_AP had an interaction effect between the shape of the BOS and the task characteristics $(p<.01)$.

CONCLUSIONS Postural control during the intervention of a cognitive task is complicated based on the characteristics and difficulty of the task. The reduced movement of linear variables and the increased complexity and frequency variables when under unstable postural control (i.e., tandem standing) while simultaneously conducting dual tasks reflect the active and efficient stabilization strategies of young adults.

(c) The Asian Society of Kinesiology and the Korean Academy of Kinesiology

\section{Introduction}

Human motion is formed through the complex process of postural control combined with the joints and central nervous system, the sense organs, and biomechanical components [1]. Postural control is a complex mechanism in which multiple sensory (visual, vestibular, and somatosensory) information

*Correspondence: Sunghoon Shin, School of Kinesiology, Yeungnam University, Daehakro 280, Gyeongsan, South Korea; Tel: +82-10-8940-2406; Fax: +82-53-8104669; E-mail: sshin27@ynu.ac.kr and muscular activities act simultaneously [2]. Posture control is defined as the ability to maintain or restore the body's position in space with the goal of movement or balance [3], which is seen as an inevitable process of movement [4]. In particular, a lack of posture controllability was identified as an important internal component of a fall risk [5]. There is evidence that the combination of several causes of postural control loss increases the risk of a fall. For example, damage to the proprioceptive or vestibular system dampens the stability 
and equilibrium of the posture [6] and slows the response rate from external stimuli [7]. Damage to both the central nervous system and the peripheral nervous system [8], along with leg weakness [9], decreased the ability to achieve balance. In addition, the shape and size of the BOS during postural control in biomechanical terms are known to influence one's balance ability [7]. In general, a method for effectively modifying the shape and size of the BOS is considered to be an important balancing strategy to ensure posture stability [10]. In a previous study evaluating the balance ability of static posture control for adults aged 30 years and older, Era et al. [11] reported that, when the shape of the BOS is in a polygon form and is out of left and right symmetrical balance, the posture stability in a static upright posture is lost. Amiridis et al. [12] reported that a posture in which the two feet are vertically aligned in the anteroposterior direction (i.e., a tandem stance), as compared with a static upright posture in which the feet are symmetrical in the mediolateral direction (i.e., a normal stance), exhibits a larger COP displacement. Therefore, as an important consideration in ensuring the stability of posture control, both the size and shape of the BOS should be considered as important factors [7]. These two factors are closely related to the dynamic stability found in complex motion control strategies [13].

With regard to the effects of cognitive function on postural control, a dual-task paradigm has been applied, which requires cognitive function to evaluate the postural stability [4]. Simultaneous work of the central nervous system is known to cause instability of posture control because the two tasks must compete or share a limited cognitive domain $[14,15]$. In a previous study by Granacher et al. [16], the elderly show greater COP displacement for inverse calculations requiring more cognitive function, as compared to single tasks. In addition, Pellecchia [17] reported the greatest variation in the anteroposterior movement of a static standing posture during an inverted task when compared to a single task for young adults. This suggests that a dual task can be applied to assess in-depth the degree of prediction of falls, as well as the postural stability during complex daily activities, rather than using measurements conducted under a single task [18].

There are two traditional models for dual tasks, namely, the limited attention model and the U-shape nonlinear interaction model [19]. For example, in the limited attention model, an easy secondary dual task may not affect the posture stability, but a difficult secondary dual task may do so owing to the split-attention effect (posture control and secondary task) when performing both tasks simultaneously [20]. In the U-shape model, an upright posture may be better or worse based on the nature of the secondary task. Performing an easy secondary task in an upright posture improves the posture control without focus on the posture stability. However, when cognitive tasks are difficult, attitude control consistent with a model of limited attention resources will be exacerbated [21].

In general, a method for measuring the variability of the pressure center displacement, velocity, and area (95\% ellipse area) in the forward, backward, and left and right directions is mainly used by a conventional COP analysis method as measured through the ground reaction force However, in recent studies, it was found that the inherent information characteristics (i.e., complexity) of the human control system are embedded in the fluctuations of the COP of the attitude control, as well as in a linear measurement method [2224]. Zhou et al. [25] reported that, compared to traditional metrics, the degree of multi-dimensional complexity involved in standing postural changes (particularly in dual-working conditions) is a better predictor of future falls in the elderly.

In summary, in a static posture control measurement, instability in the sense of balance was shown according to the shape of the BOS, and in dual tasks requiring cognitive function, the change in postural fluctuation was larger when compared with a single task. The higher the level of difficulty one of the two tasks is, the lower the performance of the other task [26]. Dault et al. [27] reported that, as the posture becomes more difficult, the effect of the simultaneous cognitive function increases, and that, the greater the interventional demand, the greater the change in static postural control performance. Recent research has shown that the greater the difficulty of the task in terms of interaction, the less the body sways (19). That is, different effects can be expected depending on the manner in which the two variables are combined. In particular, there is a significant possibility that the posture control will change according to the shape of the BOS, similar to a task 
requiring cognitive function, such as in recent studies on lions. However, there have been no studies on the interactions between the BOS and dual tasks, which can occur frequently in everyday life. In addition, there has been a limitation in judging whether existing studies have had an interactive effect related to dual tasks owing to the linear variable-based analysis method applied. Therefore, the purpose of this study is to clarify the effects of dual tasks and their characteristics on the balance ability using multiple analysis methods. We expected that a sway movement would be smaller in semi-tandem and tandem stances, which means that young adults concentrate more on postural control, which can potentially reduce the sway movements. Specifically, based on this expectation, the following hypotheses were made: (a) dual tasks will create less sway as the posture becomes uncomfortable (i.e., normal, semi-tandem, and tandem stances in order) owing to the concentration effect, and (b) dual tasks will affect the postural dynamics differently with more complex movement patterns as compared to single tasks.

\section{Methods}

\section{Subjects}

Twenty-two young adults (11 males, 11 females) without mental or physical disorders for the past 6 months participated in this study. The number of subjects in the study was determined using the G power 3.1.3 program (Germany), with a power of 0.8 , an effect size of 0.25 , and a significance level of 0.05 . A sample number of more than 18 subjects surveyed was deemed to be a necessary component. The effect size of ANOVA with two within factors 0.25 was calculated by the partial eta-squared $\left(\eta_{p}^{2}\right), 0.06$ which was moderate level. Twenty-two subjects who agreed to participate in the experiment were included in the study. The selection criteria of the study were as follows.

(1) College students, 20 years of age

(2) Those without ankle sprains, or neck, knee, waist, or other issues

(3) Those with no visual, vestibular, or neurological problems

(4) Those with no prior experience in balance testing

The exclusion criteria of the study were as follows.

(1) Those with neurological diseases

(2) Those with musculoskeletal disorders

The researchers provided the participants with sufficient explanations regarding the experiment in advance, and the participants signed an agreement of consent. The physical characteristics of the participants are as follows. All procedures of the study were approved by the Yeungnam University Institutional Review Board (IRB \#7002016-A-2016-034). See Table 1.

\section{Measurements}

In the study, two force plates (Accusway, AMTI, USA), a PC (Dell, USA), and a data acquisition system (PXIe-1078, NI, USA) with one PXIe 6363 card were used. Thirty seconds of data were collected at a $100 \mathrm{~Hz}$ sampling rate for each condition. The participants were randomly placed on the two $50 \times 50 \mathrm{~cm}$ left and right force plates on the floor of the laboratory and asked to look up at a target fixed at the eye level $2.5 \mathrm{~m}$ ahead. They then performed the following tasks (Figure 1). Measurements were taken under a single task using normal, semi-tandem, and tandem stances in order, while the subjects maintained a static upright posture for a total of $30 \mathrm{~s}$ $[11,28]$. In general, a normal stance is the most stable form of static posture control [29]. In a semi-tandem stance, which is considered moderately difficult, one foot is ahead of the other,

Table 1. Subject demographics

\begin{tabular}{cccccc}
\hline Subjects & Age(yrs) & Height $(\mathbf{c m})$ & Weight(kg) & School yr & Cognitive score \\
\hline Male $(\mathrm{n}=11)$ & $22.36 \pm 1.02$ & $175.54 \pm 5.44$ & $70.43 \pm 7.90$ & $15.58 \pm 8.40$ & $9.00 \pm 3.36$ \\
Female $(\mathrm{n}=11)$ & $21.18 \pm 0.75$ & $163.54 \pm 6.78$ & $56.40 \pm 5.88$ & $14.93 \pm 5.605$. & $8.21 \pm 3.57$ \\
Total $(\mathrm{n}=22)$ & $21.77 \pm 1.06$ & $169.54 \pm 8.58$ & $63.42 \pm 9.88$ & $15.12 \pm 8.25$ & $8.60 \pm 3.41$ \\
\hline
\end{tabular}


and a tandem stance, which is an in-line posture back to back, is considered the most challenging element in static posture control measurements owing to its unstable form [28, 29]. During the dual tasks, the same protocol as above, along with the additional task of adding three randomly selected threedigit numbers (between 300 and 900) and subtracting by 7 , was conducted [30]. The subjects cumulatively added the three numbers and thus could barely hear the experimenter, which avoided directly affecting their sense of physical balance. Incorrectly calculated figures were not included. To avoid the influence of each condition on the BOS, the experiment was carried out randomly a total of six times, depending on the inclusion of the dual tasks.
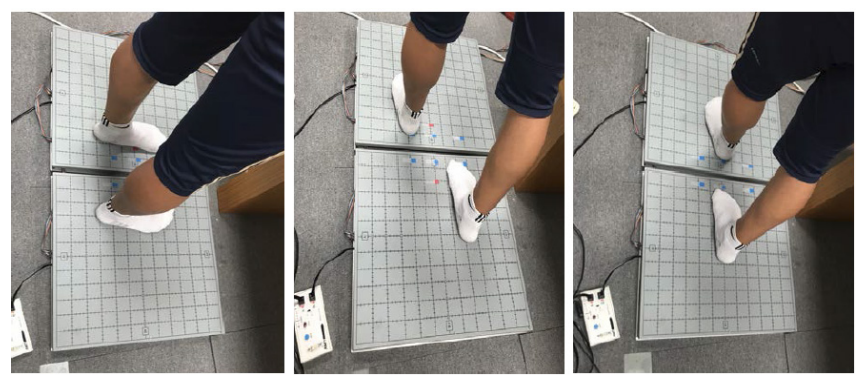

Figure 1. The shape of the base of support

\section{Data Analysis}

For the independent variables, the shape of the BOS (i.e., normal, semi-tandem, and tandem stances), and the presence or absence of dual tasks, were both established. For the dependent variables, the linear parameters (RMS, mean velocity (MV), and 95\% ellipse area (CEA)), frequency parameters (95\% power frequency (F95) and mean frequency $(\mathrm{MF})$ ), and nonlinear parameters (sample entropy (SampEn) and fractal dimension (FD)) were set in the anteroposterior and mediolateral directions [31]. In particular, the sample entropy is a representative nonlinear analysis of the same was conducted using the entropy concept [32]. To calculate the sample entropy, from the NCOP data, (N-m +1 ) vector sequences having $\mathrm{m}$ data are generated. Here, $\mathrm{m}$ represents the embedding dimension. The distance d (u (i), $u(j)$ ) between the i-th vector $u(i)$ and $j$-th vector $u(j)$ among the generated vector sequences is calculated. The probability that two or more different vectors exist is calculated using equation (1).
(1)

$C_{i}^{m}(r)=\left\{\begin{array}{c}\text { the number of } j, j \neq i, j<N-m+1, \\ \text { such that } d(u(i), u(j))<r\end{array}\right\} / N-m+1$

The sample entropy (SampEn) is calculated by substituting into Eq. (2).

(2)

SampEn $=-\ln \left[\Phi^{m}(r) / \Phi^{m+1}(r)\right]$ where $\Phi^{m}(r)=(N-m+1)^{-1} \sum_{i=1}^{N+m-1} C_{i}^{m}(r)$

FD was proposed by Higuchi [33]. First, the data interval is represented by k new time-series data. That is, the COP data existing in $k$ intervals starting from the m-th COP interval data are denoted as $X_{m}^{k}$ :

(3)

$X_{m}^{k}=\left\{\mathrm{COP}(\mathrm{m}), \operatorname{COP}(\mathrm{m}+\mathrm{k}), \operatorname{COP}(\mathrm{m}+2 \mathrm{k}), \ldots \operatorname{COP}\left(m+\left\lfloor\frac{N-m}{k}\right\rfloor k\right)\right.$

for $\mathrm{m}=1,2, \ldots, \mathrm{k}$.

In Eq. (3), $\mathrm{N}$ is the total number of COP interval data, and is the same as a round-down operation, representing only integer values of $\mathrm{a}$. The $\mathrm{k}$ pieces of time-series data calculated through the above equation are determined using Eq. (4).

(4) $\mathrm{L}_{m}(\mathrm{k})=\frac{\sum_{i=1}^{\lfloor a\rfloor}|X(m+i k)-X(m+(i-1) k)|(N-1)}{\lfloor a\rfloor \mathrm{k}}$ where $\lfloor a\rfloor=\left\lfloor\frac{N-m}{k}\right\rfloor$

When k curve lengths are calculated using Eq. (4), a graph with $\ln (1 / \mathrm{k})$ as the $\mathrm{x}$-axis and $\ln (\mathrm{Lm}(\mathrm{k}))$ as the $\mathrm{y}$-axis is generated. In the generated graph, the best fit is obtained using the least squares method. After calculating the equation, we can calculate the FD by calculating its slope. In this study, $\mathrm{k}=$ $5(1 \leq \mathrm{k} \leq 6)$ is used. A large fractal dimension means that the structure of the signal is complex. All data were analyzed using Excel (Microsoft, Inc., USA) and MATLAB (R2018a) software. A statistical analysis was conducted at a .05 significance level by performing a two-way repeated measures ANOVA with two within factors. When an interaction effect was found in the BOS type and dual tasks, a paired t-test was conducted, and all statistical processing was applied using SPSS (ver. 20). 
The effective sizes for the ANOVA are expressed as partial eta-squared $\left(\eta_{p}^{2}\right)$. The values of $\eta_{p}^{2}$ of $0.01,0.06$, and 0.14 indicate small, moderate, and large effects, respectively. The effective sizes are expressed as differences in mean as Cohen's $\mathrm{d}$ [34]. Values of Cohen's $\mathrm{d}$ of $0.2,0.5$, and 0.8 , indicate small, moderate, and large effects, respectively.

\section{Results}

The two-way ANOVA results of the linear, power frequency, and nonlinear parameters of the integrated COP obtained from two left and right ground reaction forces and moment are as follows. Figure 2 shows the sample COP trajectories under a total of six conditions for each participant.

\section{Linear parameters}

Table 2 shows the summarized results of the two-way repeated measures ANOVA with two within factors for the linear parameters. RMS_ML, MV_AP, and MV_ML showed the main effects according to the shape of the bases $(\mathrm{p}<.001)$. The more difficult the posture performed, the greater the value of each variable that was found. However, RMS_ML, MV_AP, and MV_ML did not show a main effect with dual tasks ( $p>05$ ). RMS_AP and CEA had an interaction effect on the shape of the BOS and the presence of dual tasks $(\mathrm{p}<.05)$. As a result of the posthoc test(Table 5), there was no significant difference found at the normal stance and semi-tandem stance, when two tasks(non-dual and dual) were conducted ( $\mathrm{p}>$.05). In a tandem stance, the value of the RMS_AP and CEA decreased $(\mathrm{p}<.05, \mathrm{~d}=0.88$, and $\mathrm{p}<.05, \mathrm{~d}=0.75$ respectively). RMS_ML, MV_AP, and MV_ML did not show any interaction effect ( $p>05)$.

(A) Shape of the base of support (COP) during nodual tasks

Nodual-Normal stance(COP)

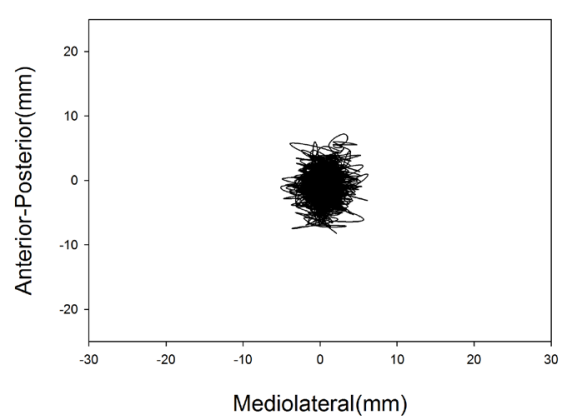

(B) Shape of the base of support (COP) during dual tasks

Dual-Normal stance(COP)

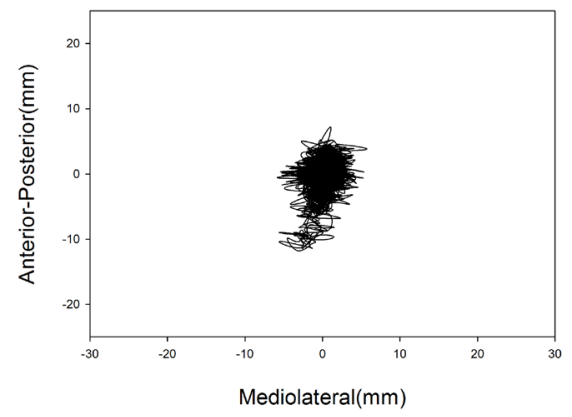

Nodual-Semi tandem(COP)

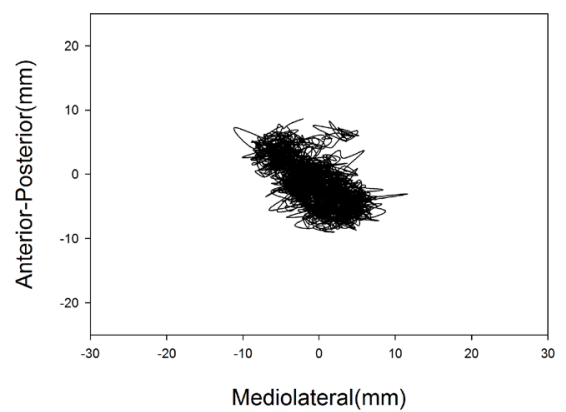

Dual-Semi tandem(COP)

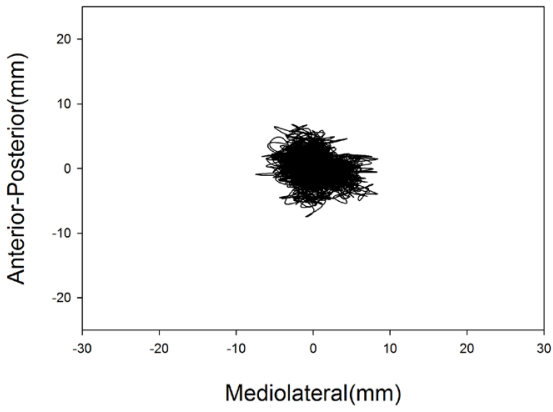

Nodual-Tandem(COP)

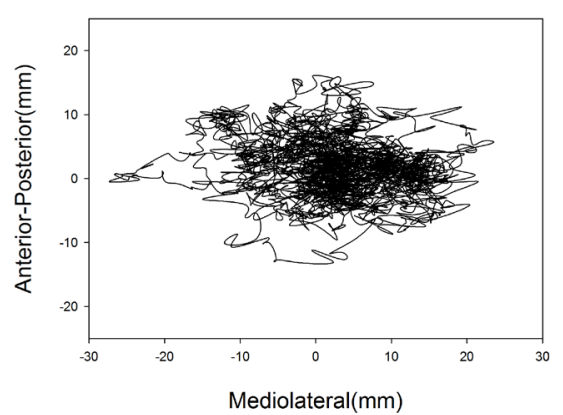

Dual-Tandem(COP)

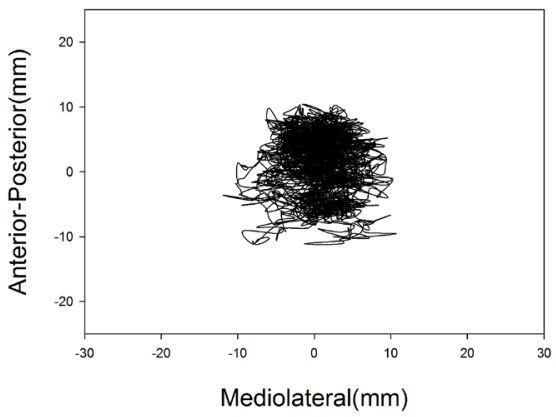

Figure 2. Shape of the base of support (COP) during nodual tasks (A) and Shape of the base of support (COP) during dual tasks (B) 


\section{Power frequency parameters}

Table 3 summarizes the results of the two-way repeated measures ANOVA with two within factors for the power frequency parameters. F95_ML and MF_ML showed the main effect according to the BOS form $(\mathrm{p}<.001)$. The more the difficult the posture that was performed, the greater the value of each variable found. In addition, F95_ML and MF_ ML did not show the main effect of the tasks with or without conducting dual tasks ( $\mathrm{p}>$.05). However, F95_AP and MF_AP had an interaction effect with the shape of the base of support and the presence of dual tasks $(\mathrm{p}<.05)$. As a result of the posthoc test(Table 5), there was no significant difference at a normal stance and a semi-tandem stance when two tasks two tasks(non-dual and dual) were performed ( $\mathrm{p}>.05)$. With the tandem stance, the value of the F95_AP and MF_AP increased sharply $(\mathrm{p}<.05, \mathrm{~d}=0.601$, and $\mathrm{p}<.05, \mathrm{~d}=0.90$ respectively). In addition, F95_ML and MF_ML did not show any interaction effect $(\mathrm{p}>.05)$.

\section{Nonlinear parameters}

Table 4 summarizes the results of the two-way repeated measures ANOVA with two within factors for the nonlinear parameters. SampEn_ML and FD_ML showed the main effect according to the BOS form $(\mathrm{p}<.001)$. For SampEn_ML, the values of each variable were increased as normal, semi-tandem, and tandem stances were conducted. FD_ML showed a larger value for each variable as normal, tandem, and semi-tandem stances were conducted. In addition, SampEn_ML and FD_ML did not show the main effects for dual tasks ( $\mathrm{p}>.05)$. However, SampEn_AP and FD_AP had an interaction effect between the shape of the BOS and the presence of dual tasks $(\mathrm{p}<.01)$. As a result of the post-hoc test(Table 5), SampEn_AP showed no significant difference at normal and semi-tandem stances when two tasks two tasks (non-dual and dual) were performed $(p>.05)$, although the value of SampEn_AP during a tandem stance was drastically increased $(\mathrm{p}<.001$, $\mathrm{d}=0.90)$. FD_AP showed no significant difference for a tandem stance $(\mathrm{P}>.05)$, but did show a significant difference at normal and semi-tandem stances $(\mathrm{p}<.05$, $\mathrm{d}=0.58$, and $\mathrm{p}<.05, \mathrm{~d}=0.38$ respectively). SampEn_ML and FD_ML did not show any interaction effects ( $\mathrm{p}>.05)$.

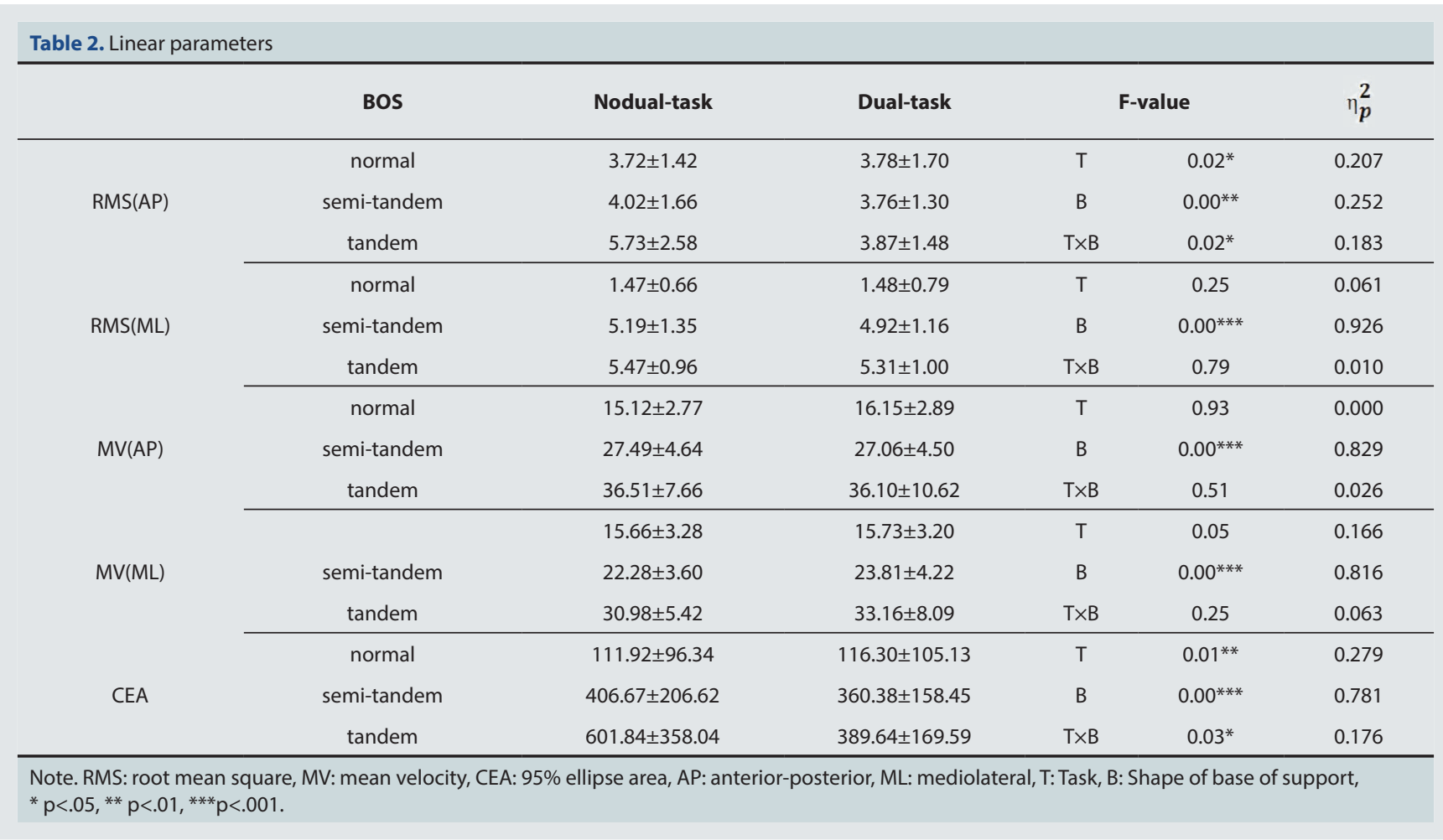


Table 3. Power frequency parameters

\begin{tabular}{|c|c|c|c|c|c|c|}
\hline \multirow[b]{2}{*}{ F95(AP) } & \multirow{2}{*}{$\begin{array}{c}\text { BOS } \\
\text { normal }\end{array}$} & \multirow{2}{*}{$\begin{array}{c}\text { Non-dual task } \\
0.89 \pm 0.36\end{array}$} & \multirow{2}{*}{$\begin{array}{c}\text { Dual-task } \\
1.00 \pm 0.35\end{array}$} & \multicolumn{2}{|c|}{ F-value } & \multirow{2}{*}{$\frac{\eta_{p}^{2}}{0.146}$} \\
\hline & & & & $\mathrm{T}$ & 0.72 & \\
\hline & semi-tandem & $2.00 \pm 0.65$ & $1.98 \pm 0.52$ & B & $0.00^{* * *}$ & 0.820 \\
\hline & tandem & $2.22 \pm 0.72$ & $2.61 \pm 0.53$ & $T \times B$ & $0.02^{*}$ & 0.164 \\
\hline \multirow[t]{3}{*}{ F95(ML) } & normal & $2.25 \pm 1.04$ & $2.19 \pm 1.05$ & $\mathrm{~T}$ & 0.48 & 0.023 \\
\hline & semi-tandem & $1.24 \pm 0.37$ & $1.39 \pm 0.49$ & B & $0.00^{* * *}$ & 0.444 \\
\hline & tandem & $1.77 \pm 0.41$ & $1.85 \pm 0.37$ & $\mathrm{~T} \times \mathrm{B}$ & 0.58 & 0.018 \\
\hline \multirow[t]{3}{*}{ MF(AP) } & normal & $0.89 \pm 0.41$ & $0.98 \pm 0.40$ & $\mathrm{~T}$ & $0.00^{* *}$ & 0.296 \\
\hline & semi-tandem & $1.49 \pm 0.48$ & $1.57 \pm 0.46$ & B & $0.00^{* * *}$ & 0.634 \\
\hline & tandem & $1.46 \pm 0.57$ & $2.04 \pm 0.69$ & $\mathrm{~T} \times \mathrm{B}$ & $0.00^{* *}$ & 0.225 \\
\hline \multirow[t]{3}{*}{$M F(M L)$} & normal & $2.53 \pm 1.27$ & $2.57 \pm 1.14$ & $\mathrm{~T}$ & 0.29 & 0.051 \\
\hline & semi-tandem & $0.91 \pm 0.31$ & $1.04 \pm 0.41$ & B & $0.00^{* * *}$ & 0.693 \\
\hline & tandem & $1.16 \pm 0.26$ & $1.24 \pm 0.26$ & $T \times B$ & 0.81 & 0.004 \\
\hline
\end{tabular}

Table 4. Nonlinear parameters

\begin{tabular}{|c|c|c|c|c|c|c|}
\hline & BOF & Non-dual task & Dual-task & \multicolumn{2}{|c|}{ F-value } & $\eta_{p}^{2}$ \\
\hline & normal & $0.84 \pm 0.34$ & $0.85 \pm 0.35$ & $T$ & $0.02^{* *}$ & 0.220 \\
\hline \multirow[t]{3}{*}{ SampEn (AP) } & semi-tandem & $1.12 \pm 0.37$ & $1.15 \pm 0.33$ & B & $0.00^{* * *}$ & 0.433 \\
\hline & tandem & $0.89 \pm 0.35$ & $1.18 \pm 0.29$ & $T \times B$ & $0.00^{* *}$ & 0.214 \\
\hline & normal & $1.83 \pm 0.37$ & $1.85 \pm 0.39$ & $\mathrm{~T}$ & 0.18 & 0.084 \\
\hline \multirow[t]{3}{*}{ SampEn (ML) } & semi-tandem & $0.64 \pm 0.25$ & $0.68 \pm 0.31$ & B & $0.00 * * *$ & 0.941 \\
\hline & tandem & $0.63 \pm 0.21$ & $0.67 \pm 0.20$ & $T \times B$ & 0.95 & 0.001 \\
\hline & normal & $1.61 \pm 0.85$ & $1.56 \pm 0.08$ & $\mathrm{~T}$ & 0.21 & 0.072 \\
\hline \multirow[t]{2}{*}{$\mathrm{FD}(\mathrm{AP})$} & semi-tandem & $1.71 \pm 0.06$ & $1.68 \pm 0.09$ & B & $0.00^{* * *}$ & 0.794 \\
\hline & tandem & $1.69 \pm 0.08$ & $1.73 \pm 0.08$ & $T \times B$ & $0.00^{* * *}$ & 0.308 \\
\hline \multirow[t]{3}{*}{$\mathrm{FD}(\mathrm{ML})$} & normal & $1.86 \pm 0.06$ & $1.84 \pm 0.10$ & T & 0.16 & 0.091 \\
\hline & semi-tandem & $1.53 \pm 0.07$ & $1.50 \pm 0.08$ & B & $0.00^{* * *}$ & 0.948 \\
\hline & tandem & $1.53 \pm 0.06$ & $1.53 \pm 0.05$ & $T \times B$ & 0.40 & 0.041 \\
\hline
\end{tabular}

\section{Discussion}

The purpose of this study was to determine how the interaction effect appears according to the combination of two variables (i.e., shape of the base of support and the presence of dual tasks).

First, we hypothesized that dual tasks would make the posture sway less as the posture became uncomfortable (i.e., in order of normal, semi-tandem, and tandem stances) owing to the concentration effect. The results showed that when the task was difficult, there was an effect of focusingon the posture control. However, this was more apparent when in a tandem stance. As a result of the analysis of linear variables, the more difficult the shape of the BOS, the larger the anteroposterior and mediolateral movements were, which is consistent with several previous studies $[11,12,28]$. These results show that a gradual change of the shape and size of the BOS according to the 
Table 5. Interaction with Base of suport and dual -task

\begin{tabular}{|c|c|c|c|c|}
\hline COP & Direction & Normal stance & Semi-tandem & Tandem \\
\hline \multirow[t]{2}{*}{ RMS } & AP & N.S & N.S & Decrease $(\mathrm{P}<.01)$ \\
\hline & ML & N.S & N.S & N.S \\
\hline \multirow[t]{2}{*}{ MV } & AP & N.S & N.S & N.S \\
\hline & $M L$ & N.S & N.S & N.S \\
\hline \multirow[t]{2}{*}{ CEA } & AP & N.S & N.S & Decrease $(P<.05)$ \\
\hline & $M L$ & N.S & N.S & N.S \\
\hline \multirow[t]{2}{*}{ F95 } & AP & N.S & N.S & Increase $(\mathrm{P}<.05)$ \\
\hline & $M L$ & N.S & N.S & N.S \\
\hline \multirow[t]{2}{*}{ MF } & AP & N.S & N.S & Increase $(\mathrm{P}<.01)$ \\
\hline & ML & N.S & N.S & N.S \\
\hline \multirow[t]{2}{*}{ SampEn } & AP & N.S & N.S & Increase $(\mathrm{P}<.001)$ \\
\hline & $M L$ & N.S & N.S & N.S \\
\hline \multirow[t]{2}{*}{$\mathrm{FD}$} & AP & Decrease $(\mathrm{P}<.05)$ & Decrease $(P<.05)$ & N.S \\
\hline & $\mathrm{ML}$ & N.S & N.S & N.S \\
\hline
\end{tabular}

Note. COP: center of pressure, RMS: root mean square, MV: mean velocity, CEA: 95\% eillipse area, F95: power frequency 95\%, MF: mean frequency, SampEn: sample entropy, FD: fractal dimension, AP: anterior-posterior, ML: mediolateral, N.S: non-significant.

difficulty in the static posture can lead to a decrease in postural control ability and weaken the balance. We also observed changes in movement strategies in the static posture control when tasks requiring cognitive function were applied. In a previous study by Pellecchia [17], three cognitive task conditions were presented (i.e., a number classification tasks, a number of list tasks, and an inverse calculation), and the difference in posture control was then examined. Of the randomly assigned three-digit numbers, when counting inversely, a larger anteroposterior movement was shown than the number sorting task. However, in this study, the differences between the RMS_ML, MV_ML, and MV_AP task variables were not clear. These results are likely to be due to differences in the task difficulty [16]. If the concurrent cognitive processing requirements are too low or too high, the postural control performance may improve or degrade [30]. For example, Riley et al. [35] reported that when performing numerical work on college students, the shaking of the static posture decreased as compared with low-level work. Therefore, this study suggests that there is a process of information integration between posture control and cognition and attention requirements that cannot be explained by a simple linear relationship depending on the task characteristics and difficulty [35-37]. In this study, we considered the interaction effect according to the difference in change of shape and the task characteristics of the BOS. Interestingly, in the linear variables (RMS_AP and CEA), the range of anteroposterior movements was reduced only during a tandem stance when dual tasks were involved. These results show that the higher the concentration of attention demanded, the more the attitude control performance improves as the difficult posture progresses during a cognitive task [38]. The most effective posture control strategy seems to have been adopted when attempting to control small but active muscle nerves simultaneously and efficiently through the performance of two relatively difficult requirements. Changes in the neuromuscular control according to shape of the base of support were described by Amiridis et al. [12]. In the study, EMG equipment was attached to the muscle surface of the hip joint to confirm the muscle activation. As a difficult posture was performed, the reliance on the individual joints was increased. The elderly reported that they rely more on their hip muscles and took a stable postural control strategy. In contrast, young adults are known to concentrate on the neuromuscular control system, which promotes an ankle strategy rather than a hip strategy. This means that postural control strategies according to 
age can be different in the posture control process, and that attitude control strategies according to age can be changed in the intervention of the concentration and cognitive processes.

Second, we hypothesized that dual tasking will affect the postural dynamics differently with more complex movement patterns as compared to single tasks. Several posthoc analyses of the interaction effects of this study indicate that a tandem stance shows decreased motion, whereas the values of the frequency variables (F95_AP and MF_AP) and complexity variable (SampEn_AP) increased. In this way, it was confirmed that the posture control of young adults shows active and efficient activation characteristics, rather than simply reducing the passive range of motion. As a similar example, Dault et al. [27] reported a decrease in the magnitude of the anteroposterior movements of the linear variables, and a significant increase in the anteroposterior direction of the frequency variable (MF_AP) when the surface condition was different and a cognitive task was involved. In fact, the complex postural control mechanism for COP change in young adults shows a variety of vibrant and active behavioral strategies, whereas less fluctuated postural control for dual tasks by the elderly is more conservative than in young adults. It can be seen that reducing the range of motion, particularly at difficult postures, and frequent changes in movement as represented by complexity and high frequency may seem to involve postural instability visually; in practice, however, this can be a stable and efficient strategy [10]. In addition, it is difficult to see that the risk of falling in a series of processes is increased because the interaction effect in the mediolateral direction is not shown, and the motion is small and complex only in the anteroposterior movements. In young adults, irregular shaking does not simply increase the risk of falling, but is rather a posture control strategy that strengthens the ankle joint to maintain proper tension in one direction as much as possible [7]. Therefore, healthy adults seem reduce the range of movements passively to maintain stable postural control when dual tasks are simultaneously performed under unstable postural control, although this takes a high concentration and active posture control strategy.

\section{Study limitations}

Although it was possible to limit the subjects to those of a similar age and educational level, it is difficult to say that the cognitive capacity between individuals has been completely controlled. In addition, although the subjects' fatigue was not reported, the repeated tests may have resulted in individual differences owing to fatigue, which could not be fully controlled. In addition, the use of a single instrument limited the analysis of each segment movement in detail. In future studies, the use of kinetic equipment such as electromyography (EMG) may help better understand the mechanism of muscle neural posture control, and thus the additional use of equipment should be attempted.

\section{Conclusions}

The conclusions of this study are as follows. First, posture control according to the intervention of a cognitive task was shown to change nonlinearly according to the task characteristics and difficulty. Second, an active and efficient stabilization strategy of young people only appears when an intentional unstable posture control is performed during dual tasks. This shows a reduced fluctuation range in linear variables, but also the characteristics of increased complexity and frequency parameters.

\section{Acknowledgments}

This research project was supported by the Sports Promotion Fund of the Seoul Olympic Sports Promotion Foundation from the Ministry of Culture, Sports, and Tourism.

\section{Conflicts of Interest:}

The authors declare no conflict of interest.

\section{References}

1. Nashner LM. Practical biomechanics and physiology of balance. Balance Function Assessment and Management. 2014; 431.

2. Horak FB, Macpherson JM. Postural orientation and 
equilibrium. Handbook of physiology. 1996; 1:255-292.

3. Pollock AS, Durward BR, Rowe PJ, Paul JP. What is balance? Clinical Rehabilitation. 2000; 14(4):402-406.

4. Granacher U, Muehlbauer T, Gruber M. A qualitative review of balance and strength performance in healthy older adults: impact for testing and training. Journal of Aging Research. 2012: 1-16.

5. Maki BE, Holliday PJ, Topper AK. A prospective study of postural balance and risk of falling in an ambulatory and independent elderly population. Journal of Gerontology. 1994; 49(2):M72-M84.

6. Shaffer SW, Harrison AL. Aging of the somatosensory system: a translational perspective. Physical Therapy. 2007; 87(2):193-207.

7. Horak FB. Postural orientation and equilibrium: what do we need to know about neural control of balance to prevent falls? Age and Ageing. 2006; 35(suppl_2):ii7-ii11.

8. Lin S-I, Woollacott MH. Postural muscle responses following changing balance threats in young, stable older, and unstable older adults. Journal of Motor Behavior. 2002; 34(1):37-44.

9. Rubenstein LZ, Josephson KR. The epidemiology of falls and syncope. Clinics in Geriatric Medicine. 2002; 18(2):141-158.

10. Shin S, Jang D. The Effect of size of base of support on variability and complexity during standing. The Korea Journal of Sports Science. 2014; 23(1):1421-1428.

11. Era P, Sainio P, Koskinen S, Haavisto P, Vaara M, Aromaa A. Postural balance in a random sample of 7,979 subjects aged 30 years and over. Gerontology. 2006; 52(4):204-213.

12. Amiridis IG, Hatzitaki V, Arabatzi F. Age-induced modifications of static postural control in humans. Neuroscience Letters. 2003; 350(3):137-140.

13. Cavanaugh JT, Guskiewicz KM, Stergiou N. A nonlinear dynamic approach for evaluating postural control. Sports Medicine. 2005; 35(11):935-950.

14. Maki BE, McIlroy WE. Cognitive demands and cortical control of human balance-recovery reactions. Journal of Neural Transmission. 2007; 114(10):1279-1296.

15. Woollacott M, Shumway-Cook A. Attention and the control of posture and gait: a review of an emerging area of research. Gait \& Posture. 2002; 16(1):1-14.

16. Granacher U, Bridenbaugh SA, Muehlbauer T, Wehrle A, Kressig RW. Age-related effects on postural control under multi-task conditions. Gerontology. 2011; 57(3):247-255.

17. Pellecchia GL. Postural sway increases with attentional demands of concurrent cognitive task. Gait \& Posture. 2003; 18(1):29-34.

18. Beauchet O, Annweiler C, Dubost V, et al. Stops walking when talking: a predictor of falls in older adults? European Journal of Neurology. 2009; 16(7):786-795.

19. Lions C, Bucci MP, Bonnet C. Postural Control Can Be Well Maintained by Healthy, Young Adults in Difficult Visual Task, Even in Sway-Referenced Dynamic Conditions. Plos One. 2016; 11(10):e0164400.

20. Wickens CD. Multiple resources and mental workload. Human Factors. 2008; .50(3):449-455

21. Wulf G, Prinz W. Directing attention to movement effects enhances learning: A review. Psychonomic Bulletin \& Review. 2001; 8(4):648-660.

22. Lipsitz LA, Goldberger AL. Loss of complexity and aging. Jama. 1992; 267(13):1806-1809.

23. Costa M, Goldberger AL, Peng C-K. Multiscale entropy analysis of complex physiologic time series. Physical Review Letters. 2002; 89(6):068102.

24. Harbourne RT, Stergiou N. Movement variability and the use of nonlinear tools: principles to guide physical therapist practice. Physical Therapy. 2009; 89(3):267-282.

25. Zhou D, Zhou J, Chen H, Manor B, Lin J, Zhang J. Effects of transcranial direct current stimulation (tDCS) on multiscale complexity of dual-task postural control in older adults. Experimental Brain Research. 2015; 233(8):2401-2409.

26. Bisgontier MP, Beets IA, Duysens J, Nieuwboer A, Krampe RT, Swinnen SP. Age-related differences in attentional cost associated with postural dual tasks: increased recruitment of generic cognitive resources in older adults. Neuroscience \& Biobehavioral Reviews. 2013; 37(8):1824-1837. 
27. Dault MC, Geurts AC, Mulder TW, Duysens J. Postural control and cognitive task performance in healthy participants while balancing on different support-surface configurations. Gait \& Posture. 2001; 14(3):248-255.

28. Wang Z, Jordan K, Newell KM. Coordination patterns of foot dynamics in the control of upright standing. Motor Control. 2012; 16(3):425-443.

29. Palmieri RM, Ingersoll CD, Stone MB, Krause BA. Centerof-pressure parameters used in the assessment of postural control. Journal of Sport Rehabilitation. 2002; 11(1):51-66.

30. Laessoe U, Hoeck HC, Simonsen O, Voigt M. Residual attentional capacity amongst young and elderly during dual and triple task walking. Human Movement Science. 2008; 27(3):496-512.

31. Mancini M, Salarian A, Carlson-Kuhta P, et al. ISway: a sensitive, valid and reliable measure of postural control. Journal of Neuroengineering and Rehabilitation. 2012; 9(1):59.

32. Richman JS, Moorman JR. Physiological time-series analysis using approximate entropy and sample entropy.
American Journal of Physiology-Heart and Circulatory Physiology. 2000; 278(6):H2039-H2049.

33. Higuchi T. Approach to an irregular time series on the basis of the fractal theory. Physica D: Nonlinear Phenomena. 1988; 31(2):277-283.

34. Cohen J. Statistical power analysis for the behavioral sciences. 2nd. Secondary. 1988, p35.

35. Riley MA, Baker AA, Schmit JM. Inverse relation between postural variability and difficulty of a concurrent shortterm memory task. Brain Research Bulletin. 2003; 62(3):191-195.

36. Vuillerme N, Nougier V. Attentional demand for regulating postural sway: the effect of expertise in gymnastics. Brain Research Bulletin. 2004; 63(2):161-165.

37. Vuillerme N, Nougier V, Teasdale N. Effects of a reaction time task on postural control in humans. Neuroscience Letters. 2000; 291(2):77-80.

38. Huxhold O, Li S-C, Schmiedek F, Lindenberger U. Dual-tasking postural control: aging and the effects of cognitive demand in conjunction with focus of attention. Brain Research Bulletin. 2006; 69(3):294-305. 